\title{
Análise multivariada da resistência do solo à penetração sob plantio direto
}

\author{
Multivariate analysis of soil resistance to penetration in no-tillage
}

\author{
Pedro Henrique Weirich Neto ${ }^{1}$ Elaine Borghi ${ }^{2}$ Claudio Bianor Sverzut ${ }^{2}$ \\ Evandro Chartuni Mantovani ${ }^{3}$ Reinaldo Lúcio Gomide ${ }^{3}$ Walter Luiz de Castro Newes $^{4}$
}

\section{RESUMO}

Com vistas ao aumento da produção, optou-se, no Brasil, pelo acréscimo de área cultivada e pelo intenso uso da motomecanização, o que acarretou prejuízos aos solos agrícolas. Paralelamente, práticas conservacionistas têm sido adotadas, a exemplo do sistema plantio direto. Em ambos os casos, estudos do estado de compactação do solo se fazem necessários. Prática e controversa, a penetrometria é a metodologia mais utilizada para o dimensionamento desse parâmetro. Assim, realizou-se trabalho em um Latossolo Vermelho sob plantio direto visando a identificar a estratificação vertical da resistência do solo à penetração. Foram amostrados 40 pontos, em malha de $50 \mathrm{~m} \times 50 \mathrm{~m}$. Em cada ponto, analisouse o comportamento do solo em três posições: na linha de semeadura, entre as linhas de semeadura e entre as linhas de semeadura com tráfego de trator agrícola. Os dados foram obtidos com penetrômetro hidráulico-eletrônico. Para identificação das camadas de solo com resistência à penetração semelhante, utilizou-se técnica de análise multivariada, denominada análise fatorial, com rotação ortogonal. Entre as linhas de semeadura, identificaram-se quatro camadas, sendo que as mais superficiais se mostraram com variância relativa maior. Nas entrelinhas com tráfego de trator e nas linhas de semeadura, locais de ação antrópica, identificaram-se três camadas, sendo as mais profundas as de variância relativa mais elevada.

Palavras-chave: compactação, índice de cone.

\section{ABSTRACT}

In order to increase agricultural production, Brazil opted for the expansion of farming areas and the intensive use of mechanization, causing serious damage to soils. At the same time, conservation practices such as no-tillage farming are also in use. Nevertheless, soil density assessments are necessary in both situations. Practical and controversial, the methodology of penetrometry is the most utilized in such assessments. The objective of this study was to identify the vertical stratification of soil resistance to penetration. Data was collected in a Typic Hapludox under no-tillage. Samples were collected in forty points of a $50 \times 50 \mathrm{~m}$ grid. Soil resistance was evaluated in three different positions in each point: plant rows, between the plant rows, and in the rows of the tractor path. Measurements were taken using a hydraulic-electronic penetrometer. Factorial analysis with orthogonal rotation, a Multivariate Analysis technique, was used to analyze soil resistance of soil layers. Four layers were identified in the plant rows, when more superficial layers had higher relative variance. Three layers were identified in the plant rows and in rows of the tractor path, points of anthropic action; in this case, deeper layers had higher relative variance.

Key words: compaction, cone index.

\section{INTRODUÇÃO}

Com a abertura de novas fronteiras agrícolas e a tecnificação da agricultura, o uso indiscriminado de máquinas e implementos agrícolas passou a ser uma constante na agricultura brasileira, provocando mudanças prejudiciais às propriedades físicas dos solos (PRIMAVESI, 1986). Uma dessas mudanças foi o aumento da densidade do solo, em conseqüência da redução do seu volume por ação de forças externas. Este processo é regido por inúmeras variáveis, além da interação destas com fatores ambientais e com o tipo de manejo do solo utilizado (JORGE, 1983).

${ }^{1}$ Laboratório de Mecanização Agrícola (Lama), Universidade Estadual de Ponta Grossa (UEPG), Av. Carlos Cavalcanti 4748, 84030-900, Ponta Grossa, PR, Brasil. Fone (42)3220-3092. E-mail: lama1@uepg.br. Autor para correspondência.

${ }^{2}$ Faculdade de Engenharia Agrícola (FEAGRI), Universidade Estadual de Campinas (UNICAMP), Campinas, SP, Brasil.

${ }^{3}$ Centro Nacional de Pesquisa em Milho e Sorgo (CNPMS), EMBRAPA, Sete Lagoas, MG, Brasil.

${ }^{4}$ Centro de Ensino e Desenvolvimento Agrícola de Florestal (CEDAF), Universidade Federal de Viçosa (UFV), Viçosa, MG, Brasil. 
Para mitigar os impactos da tecnologia no ecossistema, deve-se aprender a utilizá-la de forma a não prejudicar as propriedades do solo e o meio ambiente. Quanto ao manejo do solo, soluções têm sido apresentadas, sendo um exemplo delas o sistema plantio direto. Este tem como pilares o mínimo revolvimento do solo, a cobertura vegetal continuada do solo e a rotação de culturas. A cobertura do solo e a rotação de culturas transformaram, positivamente em função do aporte de matéria orgânica, vários processos químicos, físicos e biológicos do solo. Já o mínimo revolvimento ainda gera dúvidas quanto ao adensamento do solo, principalmente nos primeiros anos após à implantação do sistema.

Com vistas ao reconhecimento e à mensuração da compactação e do adensamento do solo, CAMARGO \& ALLEONI (1997) citam que, além dos sintomas observados nas culturas, vários métodos podem ser utilizados. BENGHOUH \& MULLINS (1990) afirmam que, através do penetrômetro, tem-se uma maneira rápida e fácil de medir a resistência à penetração do solo.

Vários autores têm correlacionado a resistência do solo à penetração com o desenvolvimento das raízes, embora MISRA et al. (1986) comentem que pelo desenho, rigidez e dimensões, esses estudos superestimam a resistência à penetração de duas a oito vezes a encontrada pelas raízes. KOOLEN \& KUIPPERS (1983) relatam a redução drástica de raízes com valores de índice de cone (IC) superiores a $3 \mathrm{MPa}$; CANARACHE (1990) sugere valores acima de 2,5 Mpa e SENE et al. (1985) consideram críticos valores de 6,0 a 7,0 MPa para solos arenosos e de 2,5 MPa para argilosos.

Estes aparelhos têm apresentado evoluções. BALASTREIRE \& AMARAL (1994) desenvolveram equipamentos com aquisição eletrônica. Já SILVA et al. (1998) e LANÇAS \& SANTOS (1998) desenvolveram equipamentos com controle de velocidade de penetração e aquisição eletrônica de sinal. WHALLEY et al. (2005) sugeriram que a haste do penetrômetro girasse.

A identificação dos limites de camadas de diferentes resistências à penetração na vertical é difícil. A maioria dos estudos é realizada com a média de valores da resistência do solo à penetração em profundidades previamente estabelecidas $(0-10 \mathrm{~cm}, 0$ $15 \mathrm{~cm}$, etc.), sem uma análise mais específica. A escolha de camadas pelo bom senso pode ser inviabilizada pela subjetividade, muitas vezes potencializada pela variabilidade dos solos, tanto na horizontal como na vertical.
É portanto comum a obtenção, em estudos visando a agricultura de precisão, de baixos valores do coeficiente de correlação espacial entre a compactação do solo e o rendimento de culturas. ALAKUKKU (1996) utilizou análise de variância para comparação e agrupamento das profundidades medidas. STELLUTI et al. (1998) utilizaram análise multivariada por componentes principais, para identificar e depois comparar a resistência à penetração das diferentes camadas. CASTRIGNAMÓ et al. (2002) representaram a variabilidade espacial da resistência do solo à penetração em três dimensões, com interpolação por análise de cokrigagem. SIRJACOBS et al. (2002) desenvolveram ferramenta pra utilização em tempo real e demonstraram correlação entre o IC e a força horizontal requerida para romper o solo. A análise multivariada, segundo MORRISON (1976), tem como objetivo o resumo de um conjunto de variáveis, considerando a variância do conjunto de dados.

O objetivo do presente estudo foi caracterizar, verticalmente, camadas com mesmo comportamento da resistência do solo à penetração com auxílio de análise multivariada de componentes principais do tipo fatorial, com rotação ortogonal.

\section{MATERIAL E MÉTODOS}

Os ensaios foram realizados em uma área de Latossolo Vermelho, na Embrapa Milho e Sorgo de Sete Lagoas, MG. Foi utilizada uma aérea com aproximadamente 38ha, manejada sob sistema plantio direto por oito anos. Por ocasião das determinações, a área estava com milho (Zea mays). A aquisição de dados foi realizada em 40 pontos localizados nas intersecções de uma malha de $50 \mathrm{~m}$ x 50m, sendo que em cada ponto foram realizados três ensaios de penetração nas seguintes posições: na linha de semeadura (LS) e na entrelinha sem e com tráfego (EST e ECT, respectivamente). O tráfego citado corresponde a uma passada do trator agrícola (Agrale 4600, com massa de $3.600 \mathrm{~kg}$ ), que acionava e transportava o penetrógrafo. Os dados foram obtidos até a profundidade de $30 \mathrm{~cm}$.

Para aquisição dos dados, utilizou-se penetrógrafo hidráulico-eletrônico desenvolvido por SILVA et al. (1998), conforme ASAE (1978). No ensaio, utilizou-se freqüência de $300 \mathrm{~Hz}$ para aquisição dos dados e velocidade de avanço do cone de $10 \mathrm{~cm} \mathrm{~s}^{-1}$ (SILVA et al., 1998). Para a análise final, calculou-se a média da resistência do solo à penetração para cada cm de profundidade. De posse destes valores, procedeu-se análise multivariada pelo método de análise fatorial, através do programa computacional Minitab, versão 12.2. 
Considerou-se cada intervalo de profundidade como uma variável independente, onde a execução da análise multivariada do tipo fatorial teria como função agrupar intervalos de profundidade (variáveis independentes) cujos valores da resistência do solo à penetração apresentassem variabilidades semelhantes. Para tal determina-se, pela análise, a correlação entre os valores das resistências do solo à penetração em cada intervalo de profundidade e um dos fatores. Quando esta correlação mostra valores elevados, interpreta-se que as camadas variavam juntas, isto é, existe a tendência de formação de uma camada. Neste caso, pode-se representar a camada por um valor médio, bem como ser utilizada para outras análises, correlações com componentes de rendimento, conteúdo de água, outros parâmetros do solo, estudos de visualizações gráficas (mapas), etc.

Para escolha dos valores de correlação representativos, deve-se considerar qual o fator em análise, pois o método considera o primeiro fator aquele em que as correlações com as variáveis (no caso, intervalo de profundidade) são mais fortes, com valores de coeficiente de correlação maiores.

O programa computacional utilizado permite a escolha do número de fatores a serem utilizados. Desta forma, escolheu-se o número cinco para todas as posições, pois, em um primeiro momento, não se sabe qual o número de fatores que representarão parte da variabilidade do fenômeno em estudo. Optou-se em demonstrar os cinco fatores para melhor visualização da análise sugerida, isto é, quando com três fatores atinge-se uma representação da variância do fenômeno, bem como uma correlação da variável com algum dos fatores, os fatores quatro e cinco também auxiliam nesta demonstração, pois os valores de correlação são baixos, e as suas variâncias relativas também assumem valores baixos.

Para facilitar a interpretação, utilizou-se o método de rotação ortogonal denominado Varimax, o qual transforma os coeficientes dos fatores de maneira que os valores das correlações entre os fatores e as $n$ variáveis originais se aproximem de zero ou de 1 (HOFFMANN, 1992). A comunalidade neste tipo de análise representa o quanto que cada variável original é explicada pelos fatores comuns.

Para simples acompanhamento e estudos futuros, determinou-se o conteúdo de água no solo nas profundidades de $0-15 \mathrm{~cm}$ e de 15 - $30 \mathrm{~cm}$, nas três posições estudadas em cada ponto amostrado.

\section{RESULTADOS E DISCUSSÃO}

A análise descritiva dos conteúdos de água no solo no momento da determinação da resistência do solo à penetração é apresentada na tabela 1 . Observa-se que, na profundidade de $15-30 \mathrm{~cm}$, o conteúdo de água tende a ser maior, bem como a variabilidade é menor, demonstrada pelo coeficiente de variação $(\mathrm{CV})$. Quanto às posições estudadas, as determinações na linha de semeadura (LS) para a profundidade de $0-15 \mathrm{~cm}$ apresentaram tendência de um menor conteúdo de água.

A resistência do solo à penetração ao longo da profundidade nas três posições de amostragem indica comportamento diferenciado para a posição de entrelinhas sem tráfego (EST) (Figura 1).

Na tabela 2, são apresentados os cinco fatores e a variância a eles atribuída, bem como a comunalidade da profundidade estudada para os valores de resistência do solo à penetração amostrada entre as linhas da cultura. Podem-se identificar quatro camadas de solo, conforme os quatro primeiros fatores. O primeiro fator (Fator 1), que representa $41,5 \%$ da variância total, demonstra claramente a existência de uma camada de 4 a $16 \mathrm{~cm}$, a qual pode ser devida à reconsolidação natural que ocorre nos solos sem aplicação de forças externas.

Tabela 1 - Análise descritiva dos conteúdos de água do solo para os pontos nas diferentes posições estudadas, no momento da determinação da resistência do solo à penetração, sob plantio direto.

\begin{tabular}{|c|c|c|c|c|c|c|}
\hline prof. & posição & média & máximo & mínimo & amplitude & \multirow{2}{*}{$\begin{array}{c}\text { CV } \\
\%\end{array}$} \\
\hline $\mathrm{cm}$ & & \multicolumn{4}{|c|}{$\mathrm{kg} \mathrm{kg}^{-1}$} & \\
\hline \multirow{3}{*}{$0-15$} & tráfego (ECT) & 0,159 & 0,362 & 0,063 & 0,299 & 30,1 \\
\hline & linha (LS) & 0,107 & 0,257 & 0,015 & 0,242 & 37,4 \\
\hline & entrelinha (EST) & 0,149 & 0,321 & 0,058 & 0,263 & 32,3 \\
\hline \multirow{3}{*}{$15-30$} & tráfego (ECT) & 0,217 & 0,359 & 0,123 & 0,236 & 18,8 \\
\hline & linha (LS) & 0,208 & 0,357 & 0,109 & 0,249 & 19,2 \\
\hline & entrelinha(EST) & 0,221 & 0,326 & 0,113 & 0,213 & 17,4 \\
\hline
\end{tabular}




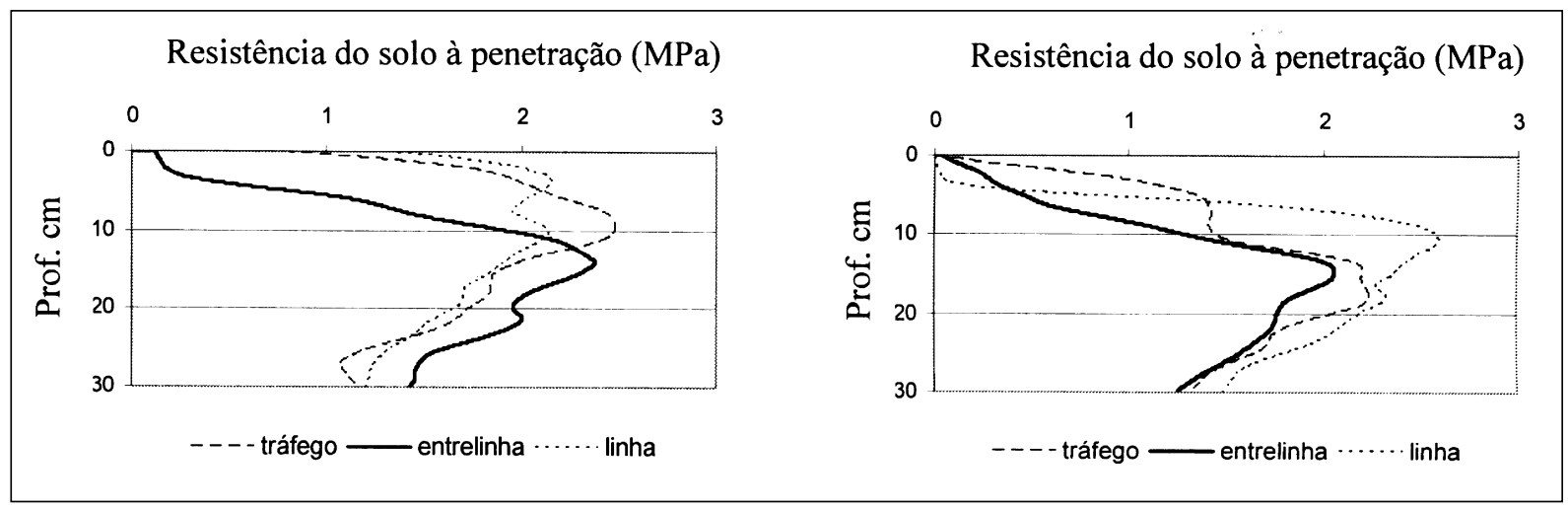

Figura 1 - Resistência do solo à penetração em dois pontos amostrais, em três posições, em solo sob plantio direto.

Tabela 2 - Coeficientes de correlação da resistência do solo à penetração em profundidades (Prof.), com cinco fatores (Fator 1, Fator 2, etc.) e comunalidade (Com.), para posição entre as linhas da cultura sem tráfego recente, com rotação ortogonal, sob plantio direto.

\begin{tabular}{|c|c|c|c|c|c|c|}
\hline $\begin{array}{l}\text { Prof. } \\
(\mathrm{cm})\end{array}$ & $\begin{array}{c}\text { Fator } \\
1\end{array}$ & $\begin{array}{c}\text { Fator } \\
2\end{array}$ & $\begin{array}{c}\text { Fator } \\
3\end{array}$ & $\begin{array}{c}\text { Fator } \\
4\end{array}$ & $\begin{array}{c}\text { Fator } \\
5\end{array}$ & Com. \\
\hline $0-1$ & 0,022 & $-0,065$ & 0,026 & $-0,941$ & $-0,118$ & 0,906 \\
\hline $1-2$ & 0,252 & $-0,081$ & 0,003 & $-0,955$ & 0,014 & 0,983 \\
\hline $2-3$ & 0,407 & $-0,088$ & 0,026 & $-0,892$ & 0,070 & 0,974 \\
\hline $3-4$ & 0,611 & -.0045 & 0,102 & $-0,732$ & 0,131 & 0,939 \\
\hline $4-5$ & 0,713 & 0,051 & 0,196 & $-0,575$ & 0,149 & 0,932 \\
\hline $5-6$ & 0,811 & 0,112 & 0,286 & $-0,404$ & 0,108 & 0,927 \\
\hline $6-7$ & 0,868 & 0,105 & 0,273 & $-0,269$ & 0,043 & 0,913 \\
\hline $7-8$ & 0,905 & 0,100 & 0,253 & $-0,236$ & 0,019 & 0,949 \\
\hline 8-9 & 0,909 & 0,133 & 0,276 & $-0,215$ & $-0,001$ & 0,966 \\
\hline $9-10$ & 0,898 & 0,175 & 0,281 & $-0,198$ & $-0,057$ & 0,955 \\
\hline $10-11$ & 0,900 & 0,201 & 0,271 & $-0,178$ & $-0,102$ & 0,966 \\
\hline $11-12$ & 0,907 & 0,222 & 0,231 & $-0,158$ & $-0,105$ & 0,980 \\
\hline $12-13$ & 0,913 & 0,239 & 0,193 & $-0,138$ & $-0,119$ & 0,960 \\
\hline $13-14$ & 0,882 & 0,286 & 0,238 & $-0,126$ & $-0,051$ & 0,934 \\
\hline $14-15$ & 0,839 & 0,305 & 0,346 & $-0,097$ & 0,032 & 0,928 \\
\hline $15-16$ & 0,805 & 0,285 & 0,467 & $-0,060$ & 0,056 & 0,954 \\
\hline $16-17$ & 0,717 & 0,281 & 0,602 & $-0,052$ & 0,117 & 0,972 \\
\hline $17-18$ & 0,656 & 0,287 & 0,667 & $-0,066$ & 0,151 & 0,984 \\
\hline $18-19$ & 0,632 & 0,307 & 0,691 & $-0,089$ & 0,115 & 0,989 \\
\hline $19-20$ & 0,593 & 0,333 & 0,713 & $-0,106$ & 0,066 & 0,987 \\
\hline $20-21$ & 0,551 & 0,353 & 0,738 & $-0,106$ & 0,023 & 0,984 \\
\hline $21-22$ & 0,517 & 0,387 & 0,743 & $-0,076$ & $-0,055$ & 0,978 \\
\hline $22-23$ & 0,442 & 0,479 & 0,724 & $-0,042$ & $-0,155$ & 0,974 \\
\hline $23-24$ & 0,352 & 0,620 & 0,641 & 0,013 & $-0,250$ & 0,981 \\
\hline $24-25$ & 0,275 & 0,736 & 0,517 & 0,089 & $-0,299$ & 0,981 \\
\hline $25-26$ & 0,232 & 0,826 & 0,404 & 0,112 & $-0,265$ & 0,981 \\
\hline $26-27$ & 0,174 & 0,914 & 0,280 & 0,118 & $-0,165$ & 0,985 \\
\hline $27-28$ & 0,176 & 0,962 & 0,173 & 0,085 & 0,001 & 0,994 \\
\hline $28-29$ & 0,167 & 0,958 & 0,132 & 0,011 & 0,167 & 0,991 \\
\hline $29-30$ & 0,156 & 0,911 & 0,111 & $-0,066$ & 0,314 & 0,970 \\
\hline Variância & 12,441 & 6,490 & 5,337 & 4,033 & 0,575 & 28,917 \\
\hline $\begin{array}{l}\text { Variância } \\
\text { relativa }\end{array}$ & 0,415 & 0,216 & 0,179 & 0,134 & 0,019 & 0,964 \\
\hline
\end{tabular}

Com 21,6\% da variância, o segundo fator caracteriza a existência de uma camada de 24 a $30 \mathrm{~cm}$, profundidade onde o preparo do solo utilizado anteriormente tende a não perturbar, isto é, a caracterização dessa camada é dada por fenômenos pedogenéticos.

O terceiro fator, que representa uma variância de $17,9 \%$ do total, valor menor, porém próximo da variância relativa do segundo fator, define uma camada de 16 a $24 \mathrm{~cm}$. Neste caso, o manejo anteriormente utilizado pode ter influenciado de maneira direta, ou ainda ser um efeito residual seu, bem como pode ser influência de uma zona de transição entre horizonte Ae B do solo, característica dos Latossolos, solos profundos e bem formados.

O quarto fator caracteriza uma camada superficial, de 0 a $4 \mathrm{~cm}$, onde, no plantio direto, dão-se as maiores transformações e, portanto, a maior variabilidade espacial, devido à intensa atividade biológica proporcionada pela cobertura vegetal morta. Neste caso, deve-se considerar também a limitação do penetrômetro, pois existe uma correlação entre a profundidade de leitura do penetrômetro e a altura do cone. Outra hipótese seria a mobilização do solo pelos mecanismos sulcadores das semeadoras em anos anteriores. O fator número cinco, pela reduzida variância relativa e pelos baixos coeficientes de correlação, não será discutido.

Os valores de resistência à penetração amostrada na linha de semeadura definem três camadas de solo, como demonstram os coeficientes de correlação e a variância relativa dos três primeiros fatores (Tabela 3). O primeiro fator, que representa 39,5\% da variância, demonstra a existência de uma camada entre 17 e $30 \mathrm{~cm}$, camada esta que pode ter sua origem em processos pedogenéticos, pois não é comum o preparo do solo alcançar tal profundidade. 
Tabela 3 - Coeficientes de correlação da resistência do solo à penetração em profundidades (Prof.), com cinco fatores (Fator 1, Fator 2, etc.) e comunalidade (Com.), para posição linha de semeadura, com rotação ortogonal, sob plantio direto.

\begin{tabular}{lcccccc}
\hline Prof.(cm) & Fator & Fator & Fator & Fator & Fator & Com. \\
& 1 & 2 & 3 & 4 & 5 & \\
\hline $0-1$ & 0,200 & 0,035 & 0,882 & 0,195 & $-0,296$ & 0,900 \\
$1-2$ & 0,132 & 0,020 & 0,972 & 0,104 & $-0,086$ & 0,980 \\
$2-3$ & 0,138 & 0,122 & 0,966 & $-0,080$ & 0,062 & 0,978 \\
$3-4$ & 0,205 & 0,246 & 0,872 & $-0,289$ & 0,162 & 0,972 \\
$4-5$ & 0,215 & 0,375 & 0,752 & $-0,428$ & 0,181 & 0,968 \\
$5-6$ & 0,178 & 0,520 & 0,658 & $-0,499$ & 0,144 & 0,971 \\
$6-7$ & 0,161 & 0,646 & 0,516 & 0,513 & 0,035 & 0,974 \\
$7-8$ & 0,147 & 0,762 & 0,377 & 0,482 & $-0,065$ & 0,982 \\
$8-9$ & 0,162 & 0,868 & 0,220 & $-0,359$ & $-0,104$ & 0,976 \\
$9-10$ & 0,228 & 0,914 & 0,120 & $-0,224$ & $-0,134$ & 0,966 \\
$10-11$ & 0,259 & 0,939 & 0,066 & $-0,046$ & $-0,075$ & 0,968 \\
$11-12$ & 0,215 & 0,963 & $-0,045$ & 0,017 & 0,006 & 0,976 \\
$12-13$ & 0,230 & 0,954 & $-0,091$ & 0,058 & 0,065 & 0,978 \\
$13-14$ & 0,325 & 0,922 & $-0,088$ & 0,029 & 0,114 & 0,977 \\
$14-15$ & 0,452 & 0,854 & $-0,073$ & 0,017 & 0,174 & 0,968 \\
$15-16$ & 0,545 & 0,788 & $-0,066$ & 0,001 & 0,225 & 0,973 \\
$16-17$ & 0,651 & $-0,704$ & $-0,076$ & $-0,036$ & 0,228 & 0,979 \\
$17-18$ & 0,752 & $-0,577$ & $-0,102$ & $-0,080$ & 0,237 & 0,971 \\
$18-19$ & 0,819 & $-0,474$ & $-0,118$ & $-0,104$ & 0,227 & 0,972 \\
$19-20$ & 0,853 & $-0,432$ & $-0,124$ & $-0,106$ & 0,190 & 0,976 \\
$20-21$ & 0,865 & $-0,397$ & $-0,133$ & $-0,142$ & 0,181 & 0,976 \\
$21-22$ & 0,882 & $-0,355$ & $-0,118$ & $-0,151$ & 0,200 & 0,980 \\
$22-23$ & 0,905 & $-0,307$ & $-0,106$ & $-0,150$ & 0,178 & 0,979 \\
$23-24$ & 0,932 & $-0,238$ & $-0,091$ & $-0,161$ & 0,113 & 0,973 \\
$24-25$ & 0,954 & $-0,213$ & $-0,103$ & $-0,080$ & 0,045 & 0,974 \\
$25-26$ & 0,959 & $-0,216$ & $-0,132$ & $-0,035$ & $-0,032$ & 0,986 \\
$26-27$ & 0,948 & $-0,185$ & $-0,167$ & 0,042 & $-0,129$ & 0,979 \\
$27-28$ & 0,929 & $-0,144$ & $-0,193$ & 0,055 & $-0,224$ & 0,975 \\
$28-29$ & 0,897 & $-0,101$ & $-0,213$ & 0,101 & $-0,307$ & 0,964 \\
$29-30$ & 0,873 & $-0,068$ & $-0,209$ & 0,126 & $-0,351$ & 0,995 \\
& & & & & & \\
\hline Variância & 11,854 & 9,833 & 5,108 & 1,417 & 0,918 & 29,130 \\
Variância & 0,395 & 0,328 & 0,170 & 0,047 & 0,031 & 0,971 \\
& & & & & & \\
\hline & & & & & &
\end{tabular}

O segundo fator mostra uma camada de 6 a $17 \mathrm{~cm}$, camada esta que pode ser caracterizada pelo contato entre o sistema de abertura de sulco para a semente (neste caso, disco duplo defasado) e o solo, tendo este último servido de apoio para o sistema. FEY (2000), testando três sulcadores, alcançou valores médios da profundidade do sulco de fertilizante de 9 , 14,7 e $10,9 \mathrm{~cm}$ para sistema duplo disco, facão e guilhotina, respectivamente.

O fator número três apresentou variância relativa de $17 \%$, caracterizando uma camada superficial, de 0 a $6 \mathrm{~cm}$, onde o solo foi desestruturado pelo mecanismo sulcador para deposição da semente, dois meses antes das análises com o penetrômetro. Pelos baixos valores de variância relativa, os fatores quatro e cinco não serão discutidos.

Os valores de resistência à penetração determinada entre as linhas de semeadura da cultura, coincidente com a passagem dos pneus do trator, sugerem a presença de uma camada de 16 a $30 \mathrm{~cm}$, indicada pelo primeiro fator, que representa uma variância relativa de 39,1\%, o que também pode ser creditado a fenômenos pedogenéticos (Tabela 4).

Analisando-se em conjunto os fatores dois e três, observa-se que a variância relativa do fator três aumentou em relação às posições anteriores

Tabela 4 - Coeficientes de correlação da resistência do solo à penetração em profundidades (Prof.), com cinco fatores (Fator 1, Fator 2, etc.) e comunalidade (Com.), para posição entre as linhas de semeadura com passagem de trator agrícola, com rotação ortogonal, sob plantio direto.

\begin{tabular}{|c|c|c|c|c|c|c|}
\hline $\begin{array}{l}\text { Prof. } \\
\text { (cm) }\end{array}$ & $\begin{array}{c}\text { Fator } \\
1\end{array}$ & $\begin{array}{c}\text { Fator } \\
2\end{array}$ & $\begin{array}{c}\text { Fator } \\
3\end{array}$ & $\begin{array}{c}\text { Fator } \\
4\end{array}$ & $\begin{array}{c}\text { Fator } \\
5\end{array}$ & Com. \\
\hline $0-1$ & 0,040 & 0,006 & $-0,927$ & 0,106 & 0,173 & 0,903 \\
\hline $1-2$ & 0,121 & 0,080 & $-0,963$ & 0,078 & 0,006 & 0,955 \\
\hline $2-3$ & 0,135 & $-0,217$ & $-0,951$ & 0,099 & $-0,035$ & 0,981 \\
\hline $3-4$ & 0,156 & $-0,352$ & $-0,901$ & 0,112 & $-0,071$ & 0,977 \\
\hline $4-5$ & 0,207 & $-0,493$ & $-0,800$ & 0,089 & $-0,115$ & 0,947 \\
\hline $5-6$ & 0,309 & $-0,589$ & $-0,688$ & 0,061 & $-0,157$ & 0,944 \\
\hline $6-7$ & 0,414 & $-0,679$ & $-0,550$ & 0,060 & $-0,190$ & 0,974 \\
\hline $7-8$ & 0,471 & $-0,745$ & $-0,387$ & 0,099 & $-0,208$ & 0,979 \\
\hline $8-9$ & 0,464 & $-0,788$ & $-0,324$ & 0,158 & $-0,127$ & 0,982 \\
\hline $9-10$ & 0,424 & $-0,820$ & $-0,280$ & 0,213 & $-0,035$ & 0,978 \\
\hline $10-11$ & 0,403 & $-0,829$ & $-0,233$ & 0,266 & 0,038 & 0,977 \\
\hline $11-12$ & 0,446 & $-0,801$ & $-0,213$ & 0,297 & 0,089 & 0,983 \\
\hline $12-13$ & 0,496 & $-0,760$ & $-0,189$ & 0,337 & 0,102 & 0,983 \\
\hline $13-14$ & 0,523 & $-0,700$ & $-0,197$ & 0,415 & 0,067 & 0,980 \\
\hline $14-15$ & 0,541 & $-0,628$ & $-0,206$ & 0,502 & 0,017 & 0,981 \\
\hline $15-16$ & 0,560 & $-0,528$ & $-0,210$ & 0,587 & $-0,009$ & 0,981 \\
\hline $16-17$ & 0,601 & $-0,444$ & $-0,217$ & 0,620 & $-0,036$ & 0,991 \\
\hline $17-18$ & 0,644 & $-0,401$ & $-0,188$ & 0,611 & $-0,056$ & 0,987 \\
\hline $18-19$ & 0,661 & $-0,421$ & $-0,184$ & 0,577 & $-0,039$ & 0,982 \\
\hline $19-20$ & 0,684 & $-0,453$ & $-0,179$ & 0,523 & $-0,022$ & 0,979 \\
\hline $20-21$ & 0,744 & $-0,457$ & $-0,182$ & 0,422 & 0,067 & 0,979 \\
\hline $21-22$ & 0,817 & $-0,400$ & $-0,173$ & 0,314 & 0,151 & 0,979 \\
\hline $22-23$ & 0,883 & $-0,339$ & $-0,152$ & 0,213 & 0,147 & 0,985 \\
\hline $23-24$ & 0,924 & $-0,301$ & $-0,147$ & 0,113 & 0,103 & 0,989 \\
\hline $24-25$ & 0,939 & $-0,284$ & $-0,154$ & 0,060 & 0,050 & 0,992 \\
\hline $25-26$ & 0,934 & $-0,298$ & $-0,150$ & 0,103 & $-0,014$ & 0,994 \\
\hline $26-27$ & 0,909 & $-0,321$ & $-0,150$ & 0,172 & $-0,095$ & 0,991 \\
\hline $27-28$ & 0,866 & $-0,347$ & $-0,159$ & 0,229 & $-0,191$ & 0,984 \\
\hline $28-29$ & 0,844 & $-0,333$ & $-0,143$ & 0,266 & $-0,251$ & 0,978 \\
\hline $29-30$ & 0,820 & $-0,349$ & $-0,155$ & 0,280 & $-0,264$ & 0,966 \\
\hline Variância & 11,724 & 8,087 & 5,906 & 3,119 & 0,443 & 29,279 \\
\hline $\begin{array}{l}\text { Variância } \\
\text { relativa }\end{array}$ & 0,391 & 0,270 & 0,197 & 0,104 & 0,015 & 0,976 \\
\hline
\end{tabular}


(Tabelas 2 e 3). Como já discutido, a camada superficial é a que mais sofre interferência; porém, nesta posição, houve a passagem de um trator agrícola, ocasionando um novo arranjo entre as partículas do solo, o que pode ter levado a um aumento da variância do fator três. Devido à baixa variância, os fatores quatro e cinco não serão discutidos.

Observa-se ainda, no conjunto da análise (Tabelas 2, 3 e 4), os elevados valores de comunalidade, o que demonstra a representatividade dos fatores nas análises. Interessante salientar que houve inversão de importância (mostrada pela variância relativa) entre as camadas (representadas pelos fatores) em relação às posições com pressão externa (passagem do trator e linha de semeadura), quando em comparação com a posição sem pressão (entrelinha da cultura). No primeiro caso, as camadas mais profundas assumem resposta mais elevada, caracterizando a ocorrência de algum processo de perturbação nas camadas mais superficiais, diminuindo a variabilidade dessas, característica do sistema plantio direto.

A metodologia sugerida apresenta certo grau de subjetividade, como na escolha da magnitude do valor do coeficiente de determinação. Esta subjetividade pode provocar erros de 1, 2 talvez $3 \mathrm{~cm}$, em casos de dúvida do pesquisador com relação a qual fator atribuir o intervalo de profundidade em estudo.

No caso da metodologia tradicional ou simples escolha empírica de camadas (0 - 0cm; 0 - 15cm), comuns em trabalhos de resistência do solo à penetração, este erro pode ser bem maior, com a utilização de valores médios de resistência que não representariam o fenômeno corretamente.

\section{CONCLUSÕES}

A análise multivariada apontou grande parte da variabilidade vertical da resistência do solo à penetração, nas três posições estudadas, podendo-se assumir que os fatores representam variações dentro de uma mesma posição, caracterizando assim diferentes camadas no solo quanto à resistência do solo à penetração. Quanto às posições estudadas, houve comportamento diferenciado. Entre as linhas de semeadura, as camadas mais superficiais se mostraram com variância relativa maior, enquanto que as camadas mais profundas foram as de variâncias relativas mais elevadas, em posições em que houve algum tipo de alteração antrópica - linha de semeadura e entrelinhas de semeadura com tráfego.

\section{REFERÊNCIAS}

AMERICAN SOCIETY OF AGRICULTURAL ENGINEERS ASAE R313.1. St. Joseph, MI: Agricultural Yearbook of Standars, 1978. 2p.

ALAKUKKU, L. Persistence of soil compaction due to high axle load traffic. II - Long-term effects on the properties of fine-textured and organic soils. Soil Till Res, v.37,n.4, p.223238, 1996

BALASTREIRE, L.A.; AMARAL, J.R. Desenvolvimento e construção de um penetrógrafo eletrônico. Campinas: SBEA, 1994. 12p. (Paper n.94/4-281).

BENGHOUH, A.G.; MULLINS, C.E. Mechanical impedance to growth root: a review of experimental techiniques and root growth. Journal of soil science. London, v.41, p.341-58, 1990.

CAMARGO, O.A.; ALLEONI, L.R.F. Compactação do solo e o desenvolvimento das plantas. Piracicaba: O.A. Camargo, L.R.F. Alleoni, 1997. 132p.

CANARACHE, A. PENETR - A generalized semi-empirical model estimating soil resistance to penetration. Soil Till Res, Amsterdam, v.16, p.51-70, 1990.

CASTRIGNAMÓ, A. et al. 3-D spatial variability of soil strength and its change oven time in a durum wheat field in southern Italy. Soil Till Res, Amsterdam, v.65, p.95-108, 2002.

FEY, E. Desempenho de sulcadores utilizados em semeadora para plantio direto sob a palha, em solo argiloso com diferentes teores de água. 2000. $76 \mathrm{f}$. Dissertação (Mestrado em Engenharia Agrícola) - Universidade Estadual do Oeste do Paraná.

HOFFMANN, R. Componentes principais e análise fatorial. Piracicaba: Departamento de Economia e Sociologia Rural. Escola Superior de Agricultura "Luiz de Queiroz", Universidade de São Paulo, 1992. 25p. (Série Didática n. 76).

JORGE, J.A. Compactação do solo, causas, conseqüências e maneiras de evitar sua ocorrência. Campinas: Instituto Agronômico de Campinas, 1983. 23p. (Circular Técnica 22).

KOOLEN, A.J.; KUIPERS, H. Agricultural soil mechanics. Germany: Springer-Verlag, 1983. 242p.

LANÇAS, K.P.; SANTOS, C.A. Penetrômetro hidráulicoeletrônico equipado com DGPS para avaliação da compactação do solo. In: BALBUENA, R.H. et al. Ingeniería rural y mecanización agraria en ámbito Latinoamericano. La Plata: UNLP, 1998. 612p.

MISRA, R.K. et al. Maximum axial and radial growth pressure of plant roots. Plant and Soil, Dordrecht, v.98, p.315-326, 1986.

MORRISON, D.E. Multivariate statistical methods. New York: McGrw-Hill, 1976. 338p. 
PRIMAVESI, A. Manejo ecológico do solo. A agricultura em regiões tropicais. 9.ed. São Paulo: Livraria Nobel, 1986. 549 p.

SENE, M. et al. Relationships of soil texture and structure to corn yield response to subsoiling. Soil Sci Am J, Madison, v.49, p.422-427, 1985.

SILVA, M.L.L. et al. Melhoramento eletrônico e hidráulico de um penetrógrafo para aplicação em laboratório e campo. In: CONGRESSO BRASILEIRO DE ENGENHARIA AGRÍCOLA, 27., 1998, Poços de Caldas. Anais... Lavras: UFLA/SBEA, 1998. V.4, p.409-411, 445p.
SIRJACOBS, D. et al. On line soil mechanical resistance mapping and correlation with soil physical properties for precision agriculture. Soil Till Res, Amsterdam, v.64, p.231242, 2002.

STELLUTI, M. et al. Multivariate approach to evaluate the penetrometer resistance in different tillage systens. Soil Till Res, Amsterdam, v.46, p.145-151, 1998.

WHALLEY, W.R. et al. Use of effective stress to predict the penetrometer resistance of unsaturated agricultural soil. Soil Till Res, Amsterdam, v.84, p.18-24, 2005. 